\title{
Free-surface flow under a sluice gate of an inclined wall from deep water
}

\author{
L. H. Wiryanto ${ }^{1}$
}

(Received 10 January 2011; revised 26 September 2011)

\begin{abstract}
Nonlinear solutions of free surface flow under a sluice gate are studied. Upstream, the fluid is assumed to be infinitely deep, and the gate is at an angle to the horizontal axis. The flow emerges from the gate and produces a uniform flow far downstream. The problem is solved numerically by a boundary element method derived from an integral equation along the free surface. An analytical function is constructed, related to the upstream flow, so that the integral equation is solvable. As a result, a free surface flow with smooth detachment from the edge of the gate is obtained for relatively large upstream Froude numbers. As the Froude number decreases, a free surface with back flow near the edge of the gate occurs, until at a certain Froude number a stagnation point occurs.
\end{abstract}

http://journal.austms.org.au/ojs/index.php/ANZIAMJ/article/view/3851 gives this article, (C) Austral. Mathematical Soc. 2011. Published October 4, 2011. ISSN 1446-8735. (Print two pages per sheet of paper.) Copies of this article must not be made otherwise available on the internet; instead link directly to this URL for this article. 


\section{Contents}

1 Introduction

C793

2 Formulation

C795

3 Numerical procedure

C799

4 Results

C800

5 Conclusions

C802

References

C803

\section{Introduction}

We study a steady two dimensional irrotational flow of an ideal fluid in a domain bounded by an infinite horizontal floor, an inclined wall at an angle $\beta$ to the horizontal axis, representing a sluice gate, and a free surface, as illustrated in Figure 1a. Physically, the infinitely deep fluid flows through a slit under the inclined wall, and forms a stream with the free surface as a boundary. Far downstream the flow is uniform. When the net volume flux of the fluid approaching the slit is Q and the height of the slit measured from the floor is $\mathrm{D}$, the free surface profile is calculated near the edge of the gate.

Most sluice gate flow calculations assume the fluid is of finite depth upstream $[1,2,3,4,5,6]$. The solutions are characterised by uniform and supercritical flow far downstream, and the flow far upstream is supercritical or subcritical. A train of waves are obtained when the upstream flow is subcritical. Binder and Vanden Broeck [7] considered a problem involving multiple disturbances on the bottom of the channel and the free surface; such as a submerged obstacle, a pressure distribution or a sluice gate. They obtained solutions subject to a radiation condition; waves form near the gate 
and decay, so that the flow is uniform far upstream. However, all types of solution exhibit uniform and supercritical flow far downstream. Here, we find the same flow characteristics with an infinite fluid depth upstream. The difference with the finite depth solutions is the existence of back flow near the edge of the gate, eventually becoming a stagnation point. The free surface separates from the inclined wall at an angle of $2 \pi / 3$. This limiting case agrees with the result obtained by Vanden-Broeck and Tuck [8] who observed the free surface flow locally near a vertical wall. The problem in this paper is a generalisation of the vertical sluice gate solved by Wiryanto et al. [9].

Most of the works cited solve the problem numerically using a boundary element method. This method is also used to solve other free surface flows. Wiryanto and Tuck $[10,11]$ applied the method to free surface flow producing one and two jets. Free surface flows caused by a line sink or source were studied by Wiryanto [10] and Hocking and Forbes [13, 14]. The boundary element method is constructed from an integral equation for the hodograph complex variable corresponding to the particle velocity. After expressing the real part of the variable in terms of the imaginary part, the Cauchy integral theorem is applied. Problems using the boundary element method usually have the hodograph variable $\Omega$ satisfying the conditions of the Cauchy integral theorem, that is, analytic and $\Omega(\zeta) \rightarrow 0$ as $|\zeta| \rightarrow \infty$, where $\zeta$ is an artificial complex variable determined by a conformal mapping of the physical plane. However, we consider fluid of infinite depth upstream, so the second condition $\Omega(\zeta) \rightarrow 0$ of the Cauchy integral theorem is no longer satisfied, since the fluid velocity far upstream is radially uniform. Therefore, we construct an appropriate function, so that we can apply the Cauchy integral theorem.

The construction of the analytic function is explained in Section 2. A similar problem was studied for the case of zero gravity by Wiryanto [15], but the floor was terminated so that the flow became a waterfall, and analytical solutions are obtained. Section 3 presents the numerical procedure for solving the integral equation. Using the trapezoidal rule, we approximate the integral equation by a nonlinear system of equations, which is solved via a Newton iteration. Section 4 presents some plots of the surface profile and discusses 
the numerical results.

\section{Formulation}

We consider the steady two dimensional irrotational flow of an inviscid and incompressible fluid in a dam of infinite depth, bounded by an inclined wall as a sluice gate with slit of height $\mathrm{D}$. We choose Cartesian coordinates with the $x$-axis along the bottom and $y$-axis directed vertically upward intersecting the edge of the inclined wall. The volume flux emerging from the dam is $\mathrm{Q}$ per unit distance perpendicular to the plane of flow, and the flow is assumed to leave the edge of the gate tangentially; see Figure 1a.

We introduce a complex potential $f=\phi+i \psi$ whose derivative $d f / d z=$ $\mathfrak{u}-\mathfrak{i} v$ gives the fluid velocity at $z=x+i y$. For convenience, we work in dimensionless variables so that $\mathrm{Q}=1$ and $\mathrm{D}=1$, and require $\phi=0$ and $\psi=0$ at $z=0$. Therefore, the $\mathrm{f}$-plane is a strip of height 1 , the non-dimensional height of the slit; see Figure 1. In the flow domain,

$$
\nabla^{2} \phi=0 .
$$

The dynamic boundary condition is expressed by the Bernoulli equation

$$
\frac{1}{2} F^{2}\left(\phi_{x}^{2}+\phi_{y}^{2}\right)+y=\text { constant }
$$

along the free boundary representing hydrostatics pressure, where the Froude number

$$
\mathrm{F}=\frac{\mathrm{Q}}{\sqrt{\mathrm{gD}^{3}}},
$$

and $\mathrm{g}$ is the acceleration due to gravity. The kinematic boundary condition, along both the solid and free boundaries, is that the normal derivative of $\phi$ vanishes,

$$
\frac{\partial \phi}{\partial \bar{n}}=0 .
$$


Physically, this condition means that fluid particles on the boundary remain on the boundary.

To determine $\phi$, we first introduce a hodograph variable $\Omega=\tau-i \theta$ related to the complex velocity by

$$
\frac{\mathrm{df}}{\mathrm{dz}}=\mathrm{e}^{\Omega} .
$$

We then introduce a second artificial variable $\zeta=\xi+i \eta$, related to $f$ by

$$
f=-\frac{1}{\pi} \log \zeta .
$$

The conformal mapping (5) is obtained using Schwarz and Christoffel's theorem [16]. Downstream $J$ is mapped to $\zeta=0$ and the bottom edge of the gate $A$ is mapped to $\zeta=-1$, as shown in Figure $1 b$.

Instead of solving for $\phi$ directly, we determine the hodograph variable $\Omega$ as a function of $\zeta$, satisfying

$$
\nabla^{2} \Omega=0
$$

subject to the dynamic boundary condition (1). This boundary condition transforms to

$$
\frac{1}{2} \mathrm{~F}^{2} \mathrm{e}^{2 \tau}+\mathrm{y}=\mathrm{c}, \quad-1<\xi<0,
$$

where $\mathrm{c}$ is an unknown constant; and the kinematic boundary condition (3) becomes

$$
\theta= \begin{cases}\beta-\pi, & -\infty<\xi<-1, \\ 0, & 0<\xi<\infty .\end{cases}
$$

Here $\theta$ is unknown for $-1<\xi<0$.

A relation between $\theta$ and $\tau$ is required to determine the unknown variables. This is obtained from the Cauchy integral theorem. The complex function

$$
\chi(\zeta)=\Omega-\frac{\beta-\pi}{\pi} \log \zeta
$$




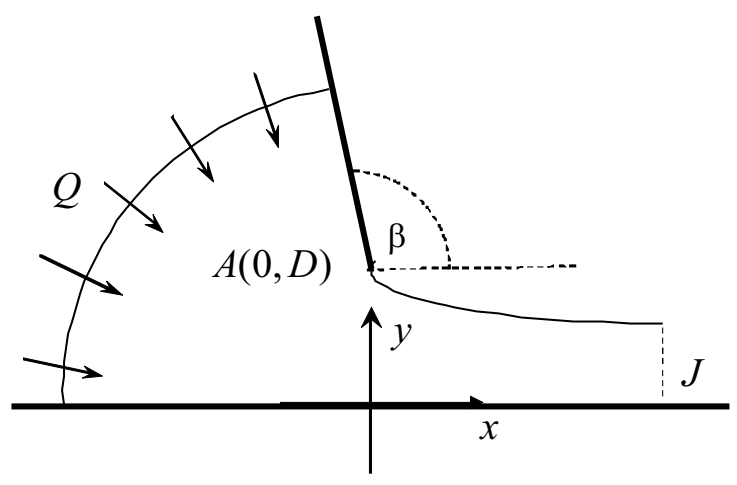

(a)
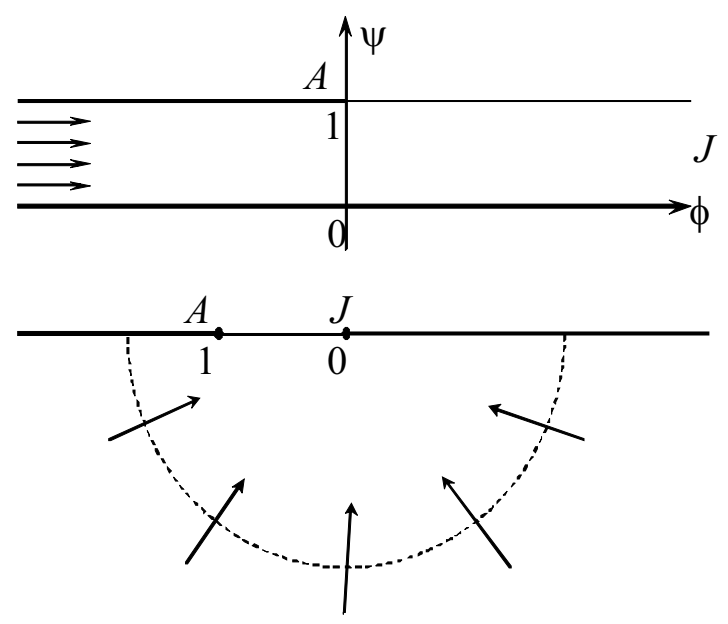

(b)

Figure 1: Sketch of the flow under a sluice gate (a) in the physical $z$-plane, (b) in the $f$-plane and the artificial $\zeta$-plane. 
is analytic and is constructed such that $\chi \rightarrow 0$ for $|\zeta| \rightarrow \infty$, so the Cauchy integral theorem can be applied. We arrive at (8) since the upstream flow far from the slit is uniform with velocity $\mathrm{df} / \mathrm{d} z \rightarrow 0$, the streamlines bounded by the horizontal floor and inclined wall having angle $\theta$ as given in (7), and the modulus of the velocity is the exponent of $\tau$. The logarithm function has the character described above, so

$$
\Omega \rightarrow \frac{\beta-\pi}{\pi} \log \zeta \quad \text { for }|\zeta| \rightarrow \infty
$$

and this is used to construct $\chi$ in (8).

Consider the closed contour consisting of the line segments from $\xi+R$ to $\xi+\delta$ and from $\xi-\delta$ to $\xi-R$, together with the semicircle $s=\xi+\delta e^{-i \alpha}$ for $0<\alpha<\pi$ and $s=\xi+R e^{i \alpha}$ for $\pi<\alpha<2 \pi$. By applying the Cauchy integral theorem to $\chi$, and then sending $\delta \rightarrow 0$ and $R \rightarrow \infty$, we obtain

$$
\chi(\xi)=-\frac{1}{i \pi} \int_{-\infty}^{\infty} \frac{\chi(s)}{s-\xi} d s \text { for }-\infty<\xi<\infty .
$$

Here, $f$ denotes a Cauchy principal value integral. The function $\chi$ is then expressed in terms of $\tau$ and $\theta$ on both sides of (9), and the real part is

$$
\tau(\xi)=\frac{\beta-\pi}{\pi} \log |1+\xi|+\frac{1}{\pi} \int_{-1}^{0} \frac{\theta}{s-\xi} \mathrm{ds} \text { for }-1<\xi<0 .
$$

The last task before obtaining the integral equation is to determine $y$ along the free surface. We use (4) and (5) to obtain

$$
\frac{\mathrm{d} z}{\mathrm{~d} \zeta}=-\frac{\mathrm{e}^{-\Omega}}{\pi \zeta}
$$

and the imaginary part along the free surface is

$$
\frac{d y}{d \xi}=-\frac{e^{-\tau} \sin \theta}{\pi \xi} .
$$


Therefore, by integrating (12),

$$
y(\xi)=1-\int_{-1}^{\xi} \frac{e^{-\tau(s)}}{\pi s} \sin \theta(s) d s
$$

where $\tau$ in (13) is evaluated from (10). The formulae for $y$ in (13) and $\tau$ in (10) are then substituted into (6), to obtain a nonlinear integral equation for $\theta$.

\section{$3 \quad$ Numerical procedure}

The nonlinear integral equation (6) converts to a set of $\mathrm{N}$ algebraic equations in $\mathrm{N}$ unknowns, if we approximate the integration (10) by summation in a suitable manner. The interval of integration $(-1,0)$ is first discretised by defining the end-points of $N-1$ subintervals $\xi_{0}=-1<\xi_{1}<\xi_{2}<\cdots<$ $\xi_{N-1}=-\epsilon$, and then we let $\theta_{j}=\theta\left(\xi_{j}\right)$ for $j=1,2, \ldots, N-1$, be the $\mathrm{N}-1$ unknowns. Here, $-\epsilon$ is a small value representing the position of the free surface relatively far from the slit, and we need this number to truncate the integration (10), as it is impossible to know the end of the free surface, but we need the subinterval $(-\epsilon, 0)$ to obtain a closed form of the equation system.

To evaluate the Cauchy principle-value singular integral in (10), we approximate $\theta(\xi)$ as varying linearly on the interval $\left(\xi_{j-1}, \xi_{j}\right)$, and evaluate the integral over each such interval exactly. For any $\xi_{j}^{*} \in\left(\xi_{j-1}, \xi_{j}\right)$,

$$
\begin{aligned}
& \tau\left(\xi_{j}^{*}\right) \approx \frac{\beta-\pi}{\pi} \log \left|1+\xi_{j}^{*}\right| \\
& +\sum_{l=1}^{N-1}\left(\theta_{l-1}-\theta_{l}\right)+\left\{\theta_{l}+\left(\theta_{l-1}-\theta_{l}\right) \frac{\xi_{j}^{*}-\xi_{l}}{\xi_{l-1}-\xi_{l}}\right\} \log \left|\frac{\xi_{l-1}-\xi_{j}^{*}}{\xi_{l}-\xi_{j}^{*}}\right| .
\end{aligned}
$$

Similarly, the integral (13) determining the $\boldsymbol{y}$-coordinate of the free surface is evaluated by numerical approximation, such as the trapezoidal rule

$$
y\left(\xi_{j}^{*}\right) \approx y\left(\xi_{j-1}^{*}\right)
$$




$$
-\frac{1}{2}\left(\frac{e^{-\tau\left(\xi_{j}^{*}\right)}}{\pi \xi_{j}^{*}} \sin \theta\left(\xi_{j}^{*}\right)+\frac{e^{-\tau\left(\xi_{j-1}^{*}\right)}}{\pi \xi_{j-1}^{*}} \sin \theta\left(\xi_{j-1}^{*}\right)\right)\left(\xi_{j}^{*}-\xi_{j-1}^{*}\right) \cdot(15)
$$

In obtaining the $N$ algebraic equations, we use $N$ collocation points $\xi_{j}^{*}$ as the mid-point in each subinterval $\left(\xi_{j-1}, \xi_{j}\right)$, except $\xi_{N}=-\epsilon / 2$ and also define $\theta\left(\xi_{j}^{*}\right)$ as the midpoint of $\theta_{j-1}$ and $\theta_{j}$. For each point $\xi_{j}^{*}$, the integral equation (6) gives one algebraic equation, so that there are $\mathrm{N}$ equations for unknowns $\theta_{1}, \theta_{2}, \ldots, \theta_{\mathrm{N}-1}$ and the constant $\mathrm{c}$ in (6). The Froude number $\mathrm{F}$ is given, and we define $\theta_{0}=-\pi / 2$ at the edge of the gate and $\theta_{\mathrm{N}}=0$ representing the uniform flow. In our computations, the nonlinear system of equations is solved by a FORTRAN subroutine, DNSQE, that does not require the user to provide a routine for the Jacobian matrix. When the iteration converges, $\mathrm{N}$-point coordinates $\left(\mathrm{x}_{\mathrm{j}}, \mathrm{y}_{\mathrm{j}}\right)$ of the free surface are determined from

$$
\frac{d x}{d \xi}=-\frac{e^{-\tau}}{\pi \xi} \cos \theta
$$

and (15) for $y$. Numerical integration is applied to (16) to get $\chi\left(\xi^{*}\right)$, using $\theta$ obtained in the previous process. We then plot the coordinates $\left(x_{\mathfrak{j}}, y_{\mathfrak{j}}\right)$ to get the surface profile.

\section{Results}

Most calculations of the numerical procedure described above use $\mathrm{N}=250$ and $\epsilon=0.000001$. These numbers are chosen as the numerical solution gives the best accuracy for the value of $\mathrm{c}$. We determine the accuracy following Wiryanto and Tuck [10]. A typical free surface for moderate Froude number is shown in Figure 2a. The flow produces a stream with a smooth free surface on leaving the inclined wall, without any waves on the free surface. The stream tends to uniform far downstream and the fluid depth is less than the height of the slit. We computed the free surface in Figure 2a for $F=0.8$ 


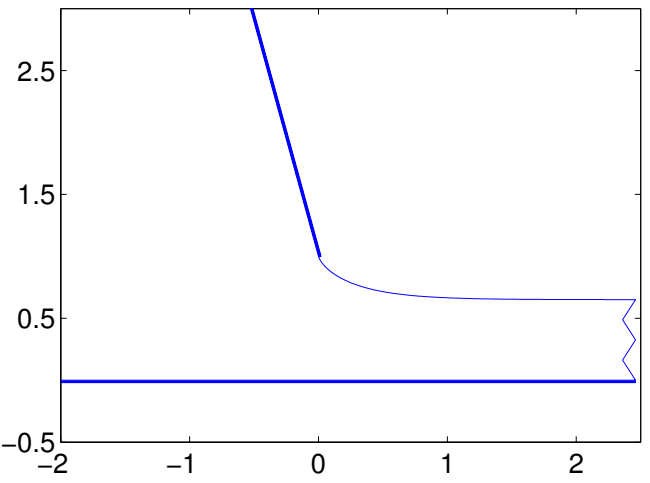

(a)

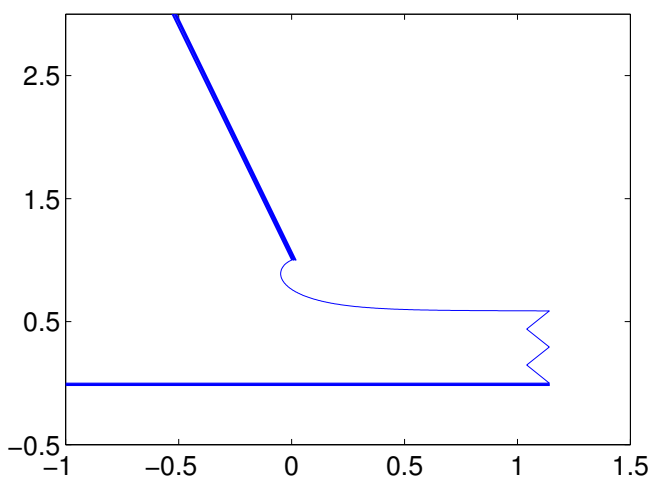

(b)

Figure 2: (a) Free surface flow under a sluice gate for $F=0.8$ and $\beta=5 \pi / 9$; (b) Free surface with a stagnation point, for the same value of $\beta$ and $F=0.225$.

and $\beta=5 \pi / 9$. For higher Froude numbers, we obtain a slightly deeper flow downstream.

For small Froude numbers $F$, we are interested in the values of $\theta$ near the edge of the gate. For $F=0.8$, we find that $\theta$ increases when increasing $\xi$, and $\theta>\beta-\pi=-1.396$, indicating the direction of the stream when separating from the gate. The $\theta$-behaviour for $F=0.4$ is similar to, but smaller than $\theta$ for $F=0.8$.

For $F<0.37$ we obtain some values of $\theta$ less than -1.396 . We define the minimum value of the curve by the coordinates $\theta_{\min }$ at $\xi_{\min }$, and we collect results from various $F$. The coordinates are plotted in Figure 3 and indicate that the stream separates from the wall smoothly, but flows increasing to the left of the separation point with decreasing $\theta_{\min }$ corresponding to a small Froude number. The position of $\theta_{\min }$ shifts, as $\theta_{\min }$ decreases, as seen in Figure 3 approaching the separation point, $\xi_{\min } \rightarrow-1$. Our numerical scheme fails for $F<0.24$ indicated by a sharp change in the $\theta$-curve near the separation point, since we define $\theta(-1)=\beta-\pi$ conflicting with the value at the next discrete point $\xi$. We extrapolate $F$ for $\theta_{\min } \rightarrow \beta-4 \pi / 3$, giving 


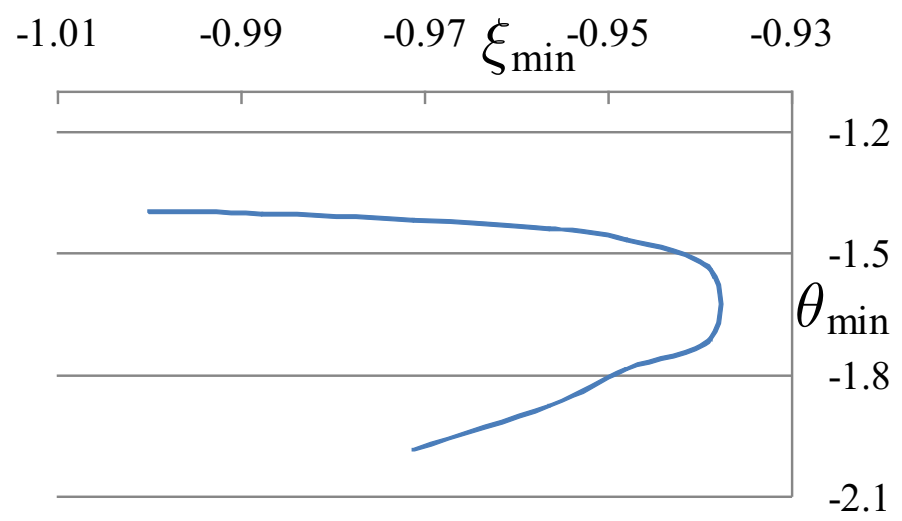

Figure 3: Plot $\theta_{\min }$ versus $\xi_{\min }$.

$\mathrm{F}=0.225$, as the limiting case, characterised by the $2 \pi / 3$ angle of the stream separating the inclined wall. The free surface flow has a stagnation point at the edge of the inclined wall. We show this limiting free surface flow in Figure 2b.

When the wall is inclined with at angle $\beta=0.38 \pi$, we calculate the free surface flow for various Froude numbers. First, we solve the problem for Froude number $F=0.25$, and obtain a smooth free surface. A free surface with back flow occurs for $F<0.25$, and a stagnation point is obtained for $F=0.13$. For smaller $\beta$, a free surface flow with a stagnation point can be obtained for smaller $F$, until $\beta=\pi / 3$, at which point the stream separates from the wall horizontally, making an angle of $2 \pi / 3$ to the inclined wall.

\section{Conclusions}

We solved numerically the free surface flow under a sluice gate from deep fluid using a boundary element method. The deep fluid upstream requires a complex analytic function constructed indirectly from the hodograph variable 
and including a term representing the character of the flow far from the slit of the gate. The resulting free surface flows without waves and with a smooth detachment at the edge of the inclined wall for the upstream Froude numbers $F>F_{0}$, where $F_{0}$ depends on the wall angle $\beta$; that is, $F_{0}=0.37$ for $\beta=5 \pi / 9$ and $F_{0}=0.25$ for $\beta=0.38 \pi$. Meanwhile solutions with back flow occur for Froude numbers smaller than $F_{0}$, and the limiting flow has a stagnation point at the edge of the gate. All types of solutions are uniform and supercritical $\left(\mathrm{F}_{\infty}>1\right)$ far downstream.

Acknowledgements I am grateful to Institut Teknologi Bandung, for supporting the research of this paper under the Research Grants, contract No. 249/K01.7/PL/2010, No. 228/I.1.C01/PL/2011, and the Research Grant from IA ITB contract No. 1443d/K01.7/PL/2010.

\section{References}

[1] D. D. Frangmeier, T. S. Strelkoff, Solution for gravity flow under a sluice gate, ASCE J. Engng. Mech. Div., 94, 1968, 153-176. C793

[2] B. E. Lorock, Gravity-affected flow from planer sluice gate, ASCE J. Engng. Mech. Div., 96, 1969, 1211-1226. C793

[3] Y. K. Chung, Solution of flow under sluice gates, ASCE J. Engng. Mech. Div., 98, 1972, 121-140. C793

[4] J. Asavanant, J.-M. Vanden-Broeck, Nonlinear free surface flows emerging from vessels and flows under a sluice gate, J. Austral. Math. Soc., 38, 1996, 63-86. doi:10.1017/S0334270000000473 C793

[5] J.-M. Vanden-Broeck, Numerical calculations of the free-surface flow under a sluice gate, J. Fluid Mech., 330, 1996, 339-347. http://journals.cambridge.org/abstract_S0022112096003849 C793 
[6] B. J. Binder, J.-M. Vanden-Broeck, Free surface flows past surfboards and sluice gates, Eur. J. Appl. Maths., 16, 2005, 601-619 doi:10.1017/S0956792505006443 C793

[7] B. J. Binder, J.-M. Vanden-Broeck, The effect of disturbances on the flows under a sluice gate and past an inclined plate, J. Fluid Mech., 576, 2007, 475-490 doi:10.1017/S0022112007004806 C793

[8] J.-M. Vanden-Broeck, E. O. Tuck, Flow near the interaction of a free surface with a vertical wall, SIAM J. Appl. Math., 54, 1994, 1-33. doi:10.1137/S0036139992240838 C794

[9] L. H. Wiryanto, J. Widjaja, B. Supriyanto, Free-surface flow under a sluice gate from deep water, Bul. Malay. Math. Soc., 34, 2011, 601-609. http://www.emis.de/journals/BMMSS/vol34_3_17.html C794

[10] L. H. Wiryanto, E. O. Tuck, A back-turning jet formed by a uniform shallow stream hitting a vertical wall, Proc. Int. Conf. on Dif. Eqs., Kluwer Academic Press, 1997. C794, C800

[11] L. H. Wiryanto, E. O. Tuck, An open-channel flow meeting a barrier and forming one or two jets, J. Austral. Math. Soc., 41, 2000, 458-472. doi:10.1017/S0334270000011760 C794

[12] L. H. Wiryanto, Small Froude number solutions of flow caused by a line source, J. Indones. Math. Soc., 6, 2000, 1-6.

[13] G. C. Hocking, L. K. Forbes, A note on the flow induced by a line sink beneath a free surface, J. Austral. Math. Soc. B, 32, 1991, 251-260. doi:10.1017/S0956792507006924 C794

[14] G. C. Hocking, L. K. Forbes, Subcritical free-surface flow caused by a line source in a fluid of finite depth, J. Eng. Math., 26, 1992, 455-466. doi:10.1007/BF00042763 C794

[15] L. H. Wiryanto, Zero gravity of a jet emerging from a slit, J. Indones. Math. Soc., 12, 2006, 89-98. C794 
[16] L. M. Milne-Thomson, Theoretical hydrodynamics. Dover Publications, Inc., 1968, 274-281. C796

\section{Author address}

1. L. H. Wiryanto, Faculty of Mathematics and Natural Sciences, Institut Teknologi Bandung, Jalan Ganesha 10 Bandung, Indonesia. mailto:leo@math.itb.ac.id 